\title{
Optical Properties of Interstellar Dust from Cosmic Dust Analogs Studied in the Lab
}

\author{
Harald Mutschke* \\ Astrophysical Institute and University Observatory, Friedrich Schiller University Jena \\ E-mail: harald.mutschkeduni-jena.de
}

In the past years, cosmic dust research has taken great advantage from the laboratory efforts to investigate the properties of analog materials such as amorphous magnesium/iron silicates and various forms of carbon. Optical properties are particularly important as they provide the link to observations and need to be incorporated into models in the form of dust opacities. Laboratory investigation of dust opacities has to cover a wide wavelength range from the UV to the millimeter wavelength range and to study the temperature dependence of the electromagnetic excitation processes governing the optical response. For understanding the underlying physics, a detailed knowledge of the microscopic structure is required, which in turn may allow predictions for dust in cosmic environments, being subject to continuous structural modifications. Given the complexity of amorphous structures, our understanding of the optical properties of interstellar dust is still incomplete. In particular, the opacity outside the strong fundamental excitations of the electron and phonon system are not yet very well constrained, but even some of the strong bands such as the $\pi-\pi^{\star}$ transition band of carbonaceous dust and its relation to the interstellar $217.5 \mathrm{~nm}$ extinction feature still require closer investigation. In this respect, it is crucial to develop further the structural analysis of such amorphous structures, and to find the relation to optical properties. This review intends to summarize the current state of these efforts.

The Life Cycle of Dust in the Universe: Observations, Theory, and Laboratory Experiments - LCDU 2013, 18-22 November 2013

Taipei, Taiwan

\footnotetext{
*Speaker.
} 


\section{Introduction}

Analog materials are a means to explore in the laboratory how cosmic dust particles may behave in the interaction with gas atoms and ions, electromagnetic radiation and fields, etc., and to find out which properties these particles need to have in order to reproduce astronomical observations. The limited information about the nature of cosmic dust particles requires studying a relatively wide range of compositions and structures. This is also needed in order to investigate the physical dependencies of observable quantities on the chemical and structural properties. Thus, dust analogs do not necessarily aim at reproducing all cosmic-dust properties, but require controllable and reproducible production techniques.

In this review, I will focus on analogs of interstellar dust, such as amorphous silicates and carbonaceous materials. Optical and spectroscopic properties of these analog materials are a primary goal to study, for providing basic data for the exploitation of observational information such as extinction, thermal emission, and scattering. Motivation to deal with the optical properties of dust analogs comes also from a number of very basic questions concerning our current understanding of dust properties and observations, such as:

- What is the source of visible/near-infrared (NIR) opacity for silicate dust? Are the GEMS (glasses with embedded metal and sulfide) interstellar dust particles?

- What is the carrier of the interstellar UV extinction hump at $217.5 \mathrm{~nm}$ wavelength?

- What is the reason for the observed anti-correlation of dust temperature and far-infrared spectral opacity index?

Answering these questions is not possible without a detailed knowledge of the relations between optical and structural properties of the dust. This review will discuss some current efforts to study experimentally such relations by means of analog materials.

\section{Laboratory analogs of ISM dust}

Highly advanced and often specifically dedicated laboratory techniques have been applied in many laboratories in order to produce interstellar dust analogs. For amorphous silicates, this

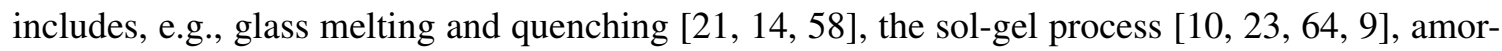

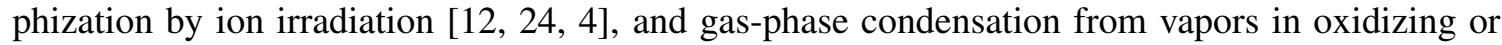
inert atmospheres [1], [7], [1, [3], [53]. Gas-phase condensation is the primary technique for making carbonaceous dust analogs, with several methods to vaporize solid carbon thermally or by laser ablation [32, 6, [6, [28]) or to introduce reactions in molecular precursors (e.g., by plasma pyrolysis [15]) or laser-induced gas pyrolysis [ [55, [35, 25]]). Bulk materials such as coals [ []], glassy carbon [B3], or pyrolysis products of solid polymers [27] have been investigated as well. Products of further processing (by ions, UV radiation, or thermal treatment) are of course separate laboratory analogs, which were often not produced to determine optical properties but to study the processes themselves.

Most production techniques are not suitable for optical studies in all spectral ranges. Transmission measurements in the regions of relative transparency (far-infrared, vis/NIR for insulators) 
require large amounts of material to detect the absorption. Consequently, millimeter-thick slabs or pellets with a high sample concentration are needed in the FIR to measure the continuum opacity, "thin sections" of tens to hundreds of micron thickness to measure impurity absorption or crystalfield bands in the visible. In the region of phonon absorption (IR bands) powders diluted by solid or liquid material or "clouds" of sub-micron grains (aerosols) can be measured in transmission, as well as sub-micron thick layers. In the range of electronic interband absorption (UV), such layers need to be one to two orders of magnitude thinner. The reflectivity of polished surfaces is a widely used alternative quantity, suitable to derive optical material data in the region of strong absorption (UV, IR). In spectral ranges of weak absorption, however, it does not deliver sufficient information to derive the (small) absorption index. This means that absorption constants in the wavelength range of the stellar radiation and of cold-dust thermal emission, which are needed for many astronomical models, remain unknown, unless analogs are available as bulk materials or at least as large amounts of powder.

In any case, cosmic dust analogs should be well characterized in terms of their structure and composition. Typical analytical methods comprise elemental analysis by X-ray spectroscopy (EDX), structural analysis by X-ray diffraction (XRD), and inspection of homogeneity and morphology by electron microscopy (SEM or TEM). For the amorphous materials used as analogs for interstellar dust, the structural analysis is particularly complex. Chemical bonding varies from site to site in an amorphous network, but its details may be important for the response to electromagnetic radiation, depending on the wavelength of the latter. Particularly for low-energetic radiation, this response will often critically depend on the temperature of the dust. In these cases, the measurement of temperature-dependent absorption gives insight into the physics of the absorption processes, but is also important for a correct interpretation of observations.

Finally, the morphology of an analog material should be considered if data are compared. Grain shape, grain size, and agglomeration influence the result of optical measurements, but data are not always available for the desired morphology. Bulk samples are a means to derive basic material data ("optical constants") from simple geometries, which can later be used in simulations of small-particle spectra using theoretical models. However, it should be noted that the result can be highly sensitive to the model if for instance the optical constants have high values (e.g., [48, 62]).

\section{UV/vis absorption of carbon and PAH mixtures}

The optical properties of interstellar carbonaceous matter are still very poorly known. While earlier dust models relied on the optical constants of graphite, the analysis of infrared absorption bands demonstrated the dominance of a hydrogenated amorphous carbon material in space [52]. In addition, polycyclic aromatic hydrocarbons, either in the form of free molecules (PAHs) or of aromatic units in "MAONs" (mixed aromatic/aliphatic organic nanoparticles [B4] ) or "arophatics" [42], are a constituent of interstellar dust, which reveals its presence by infrared emission features. Because of this complex picture that may change from one astrophysical environment to another due to interaction with, e.g., energetic radiation and shock waves, choosing the appropriate optical constants that can be used in models has always been problematic. Few sets of data have been derived so far from measurements on dust analogs over a wide wavelength range. These analogs attempt to simulate the highly variable optical properties of carbon by different hydrogen 


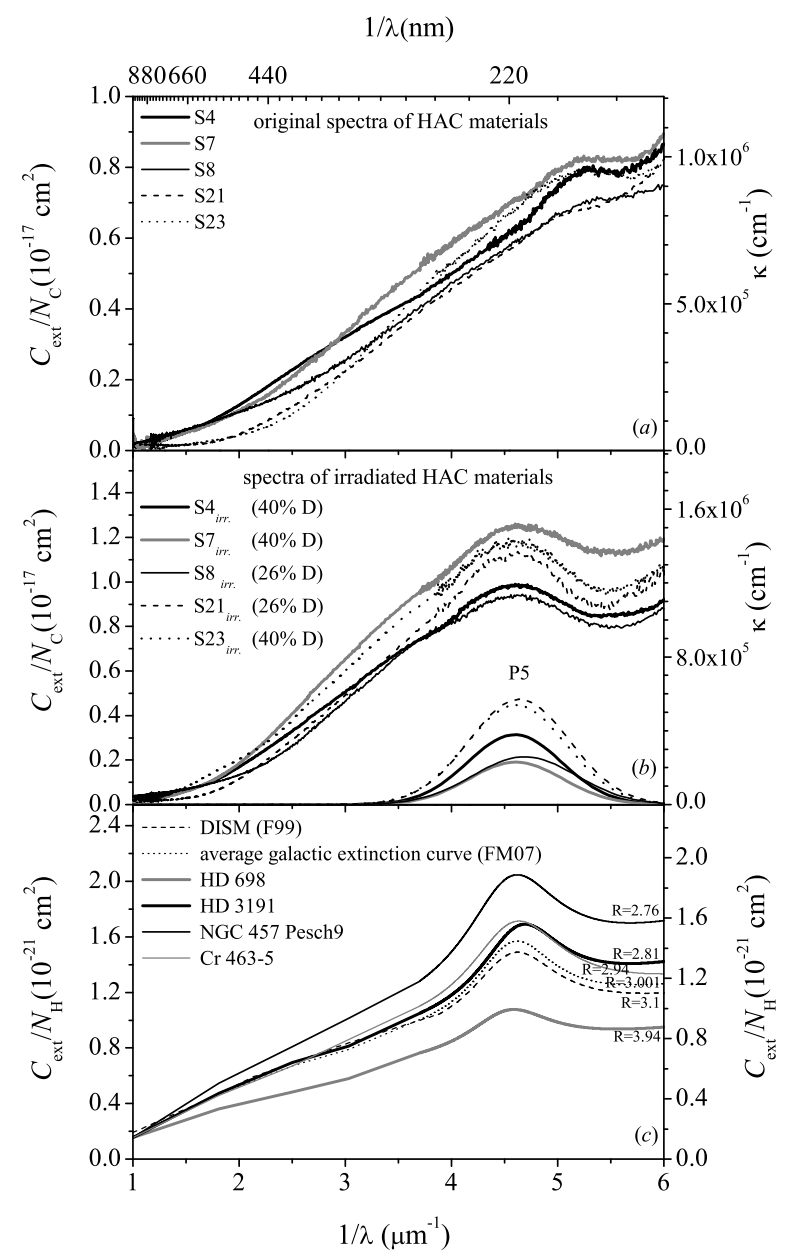

Figure 1: Extinction cross section per carbon atom $\mathrm{C}_{e x t} / \mathrm{N}_{H}$ and linear absorption coefficient $\kappa$, respectively, of thin layers of HAC particles before (a) and after (b) UV irradiation. The spectra of five samples produced at different conditions are shown. D denotes the average interstellar irradiation dose of $3 \times 10^{23} \mathrm{eV} \mathrm{cm}^{-2}$. The inset in panel $b$ shows Gaussian profiles representing the spectral peak (labeled P5) at about $217 \mathrm{~nm}$. Panel c shows interstellar extinction curves for individual objects at different $\mathrm{R}_{V}$ (the ratio of visual extinction to optical color excess [ए]] ) and averaged curves labeled F99 [ए6] and F07 [ए7]], given as extinction cross section per hydrogen atom, for comparison. From Gadallah et al. [ए8]].

concentrations in condensation experiments [68] or by different annealing temperatures in pyrolysis experiments [27]. In addition, there have been important experiments carried out to simulate the influences of UV radiation, energetic ions, reactive hydrogen gas, and thermal annealing on carbonaceous structures and spectra (e.g., [40, 37, 36] ). However, the current efforts to develop a comprehensive model of the optical properties of carbonaceous dust [BO] ] are still quite simplistic in the sense that they are based on a single physical parameter, i.e. the optical gap energy. This parameter depends primarily on the medium-range order, i.e., which is often imagined as a distribution of "graphitic" units, but high-resolution transmission electron microscopy (HRTEM) investigations show that these units, in reality, are often non-planar [28], leading to more complicated optical properties. 


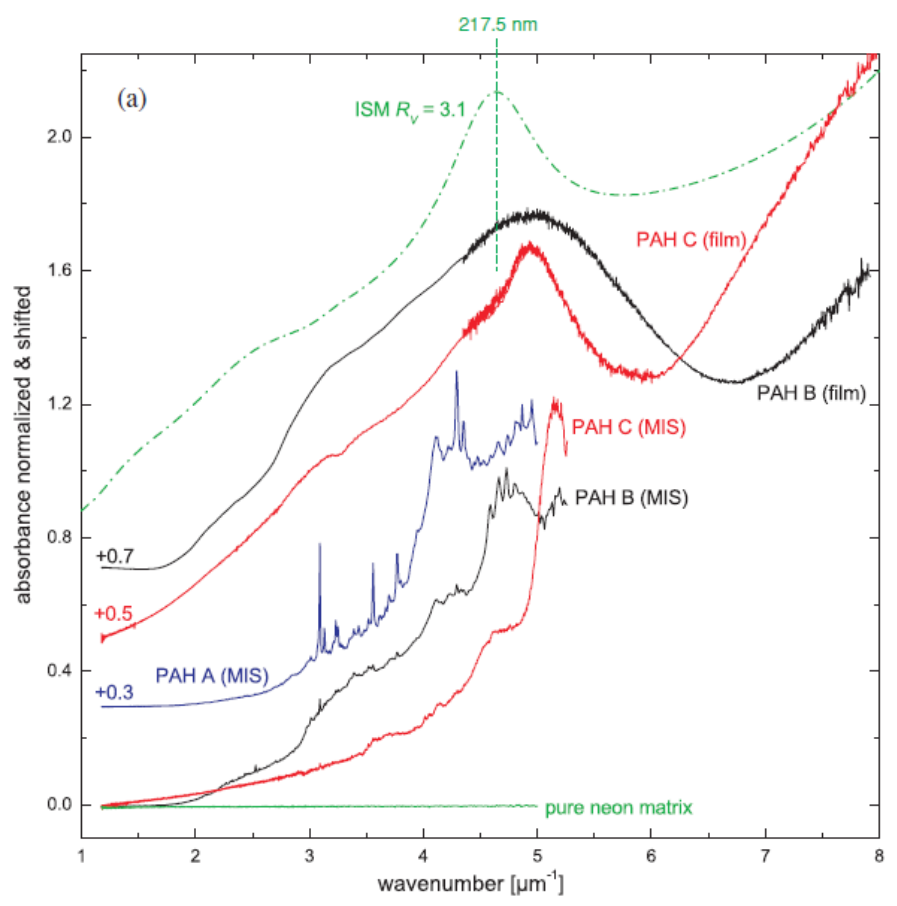

Figure 2: UV spectra of different PAH mixtures measured with the matrix isolation technique (MIS) and as compact films deposited on a $\mathrm{CaF}_{2}$ window. The mixtures have been extracted from carbonaceous analogs produced by laser-induced gas pyrolysis by using different solvents [ [26] and refined by high-pressure gas pyrolysis (sample PAH C). PAH A contains molecules with approximately 10-22 C atoms, PAH B with 10-38 C atoms, and PAH C with 22-38 C atoms. Larger molecules may be present in each mixture as well. Spectra have been shifted for clarity. Experimental results are compared with the mean interstellar extinction [B], from Steglich et al. [6]].

In this respect, an important point is that a sound reproduction of the interstellar UV extinction hump, which is widely believed to be due to $\pi-\pi^{\star}$ transitions in carbonaceous species [प5], has not yet been achieved in the laboratory. Claims that hydrogenated amorphous carbon (HAC) particles of a certain hydrogen content, if isolated from each other, possess a feature of sufficient strength and sharpness at the right wavelength position [56], have not yet been confirmed by a second experiment. These experiments suffered from an incomplete coverage of the absorption band towards shorter wavelengths, similarly as measurements of UV-irradiated HAC that showed the activation of a UV resonance at about the right spectral position [39]. Meanwhile, measurements have routinely extended into the vacuum-UV region, and the deconvolution of absorption vs. scattering losses and of $\sigma-\sigma^{\star}, \mathrm{n}-\pi^{\star}$, and $\pi-\pi^{\star}$ transitions has made progress [35], [28, [25]. Based on this, Gadallah et al. [ए8] recently performed a new UV irradiation experiment and successfully confirmed the appearance of a UV absorption feature at a wavelength of approximately $217 \mathrm{~nm}$. Although the width of this band matched approximately that of the interstellar UV hump (Fig. W), the band profile was Gaussian rather than Lorentzian and the carrier of that band could not be firmly identified in the tiny amounts of material that had been irradiated, although a considerable dehydrogenation and graphitization of the HAC material has been detected by infrared spectroscopic and electron microscopic analysis. 


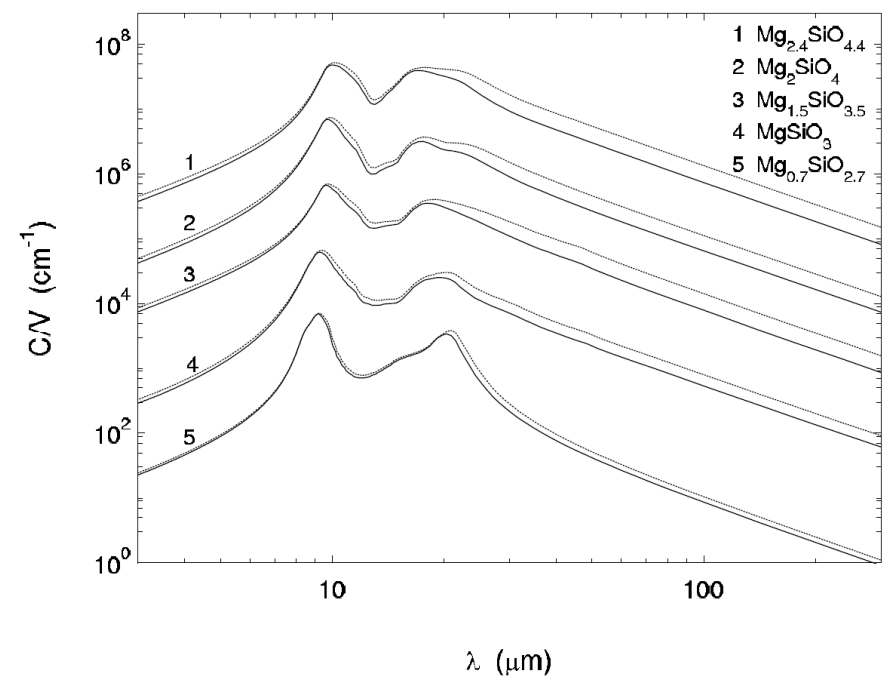

Figure 3: Absorption efficiencies of amorphous silicates with different contents of $\mathrm{Mg}$ cations in the infrared spectral range. The absorption has been calculated from experimentally determined optical constants for spherical grains (solid lines) and a distribution of ellipsoidal grains (dotted lines). From Jäger et al. [23]].

Other studies have suggested that PAH molecules contribute to the interstellar UV hump [29]. Steglich et al. [6], 59] studied mixtures of PAHs extracted from gas pyrolysis products and confirmed that a mixture of large neutral PAHs produces a strong UV absorption feature, which is, however, still at too short a wavelength for PAHs containing up to $38 \mathrm{C}$ atoms (Fig. D). Simulations predict that for larger molecules the peak would shift to larger wavelengths. Even ionization does not change the position of this peak, but could account for broadening of the feature [6]]. For a sufficiently broad mixture of molecular species, the absorption curve at longer wavelengths is also relatively smooth [59], which is an important criterion for potential carriers and has been a problem for less diverse mixtures of PAHs. It is one of the most interesting questions for the future, towards which kind of structure the two approaches of large aromatic molecules and small graphitized HAC particles will converge.

\section{Optical properties of amorphous silicates}

\subsection{Mid-infrared spectrum}

The infrared spectra of amorphous silicates as analogs of interstellar dust have been investigated since the seventies (e.g., [ㅁ] ]) and systematic studies on the influences of the cation content, and the type of cations ( $\mathrm{Mg}, \mathrm{Fe}, \mathrm{Al}, \mathrm{Ca}$ ) have been performed in the last two decades. It is now well known that in analogy to the crystalline silicates the incorporation of most cations (except for $\mathrm{Na}$, for instance) reduces the polymerization of the $\mathrm{SiO}_{4}$ tetrahedra - in other words, increases the number of non-bridging oxygens (NBOs) - and results in a shift of the Si-O stretching band towards longer wavelengths. An example is given in Fig. [1, where the band peak shifts from about $9.2 \mu \mathrm{m}$ to a wavelength larger than $10 \mu \mathrm{m}$ with increasing $\mathrm{Mg}$ content [23]]. This is usually understood as the consequence of summing up the resonance peaks of the differently polymerized $\mathrm{SiO}_{4}$ 
tetrahedra, which are located at shorter wavelengths for increasing polymerization. Interestingly, this trend is opposite for the maximum of the $20 \mu \mathrm{m}$ feature caused by Si-O bending vibrations and the vibrations of cations. Temperature does not seem to affect the position of these bands strongly [20].

However, the polymerization in an amorphous network is a statistical phenomenon that is not exclusively determined by the composition, but likely also by the process of its solidification. Spectra of amorphous silicates with the composition of, e.g., $\mathrm{MgSiO}_{3}$ therefore do not need to be identical. Independent characterization of their polymerization state would, thus, be very desirable when spectra of different analogs are compared. So far, this has been done only for few of the analogs, because the methods that are able to deliver this information are often not routinely available. The most powerful method in this respect is ${ }^{29} \mathrm{Si}$ nuclear magnetic resonance (NMR) spectroscopy, which gives relative numbers of the $\mathrm{Si}$ atoms in different structural sites by the different chemical shifts of their NMR signals. Coupeaud et al. [Q] perform such an analysis, but use the structural information primarily to interpret the far-infrared continuum absorption of their sol-gel silicates, although they also show mid-infrared spectra.

Raman spectroscopy is another, more frequently available, tool to obtain structural information. Differences between amorphous networks appear more clearly in the Raman-active vibrations than in the infrared-active ones, although the interpretation of the spectral changes is not entirely clear and the method so far has only been used by a few authors [47, 65]. Thompson et al. [65] use Raman spectroscopy and low-wavenumber X-ray scattering to detect the release of strain in medium-range order of the amorphous network during heating (at about $500^{\circ} \mathrm{C}$ ). The mid-infrared spectra were shown to be sensitive to these structural changes, reflecting the breakup of larger polymerized structures into smaller units by an increase of the longer-wavelength components of the $10 \mu \mathrm{m}$ band. Changes in the medium-range order were also observed in the X-ray absorption near edge structure (XANES) [63].

The mid-infrared spectra of Mg-Fe silicate glasses measured by Dorschner et al. [ए4] are still the only systematic study on such iron-containing amorphous silicates in the sense that the iron content has been varied systematically. These data have been used to constrain the iron content in interstellar silicates [45]. The major effect of replacing some of the magnesium with iron is a broadening of the 10 and $20 \mu \mathrm{m}$ bands [14]]. However, it is not clear how strongly this spectral behavior may be influenced by the oxidation state of the incorporated iron. While ferrous iron $\left(\mathrm{Fe}^{2+}\right)$ simply replaces magnesium as a "network modifier" cation, as it is the case for instance in olivine crystals, oxidation to ferric $\left(\mathrm{Fe}^{3+}\right)$ iron enables the iron to form new tetrahedra ("network former") and, thus, to enhance the degree of polymerization in the network. Since the iron in these glasses was partly oxidized [14], the band profile could have been affected. For these glasses, the iron oxidation state had been determined by wet chemical analysis. X-ray spectroscopy [4]] and Mössbauer spectroscopy [6] ] are other powerful techniques that should be applied when ironcontaining silicate analogs are investigated.

Aluminum is another ion that affects the state of polymerization, because it is preferentially a network former. The influence of aluminum on infrared bands has been investigated by Mutschke et al. [47] and, more recently, by Speck et al. [58]. The latter authors also synthesize an iron-free silicate of mean cosmic composition (including $\mathrm{Mg}, \mathrm{Al}, \mathrm{Na}, \mathrm{Ca}$ ) based on the claim by Min et al. [45] that interstellar silicates are poor in iron (see also [44]]). For a silicate analog of mean cosmic 


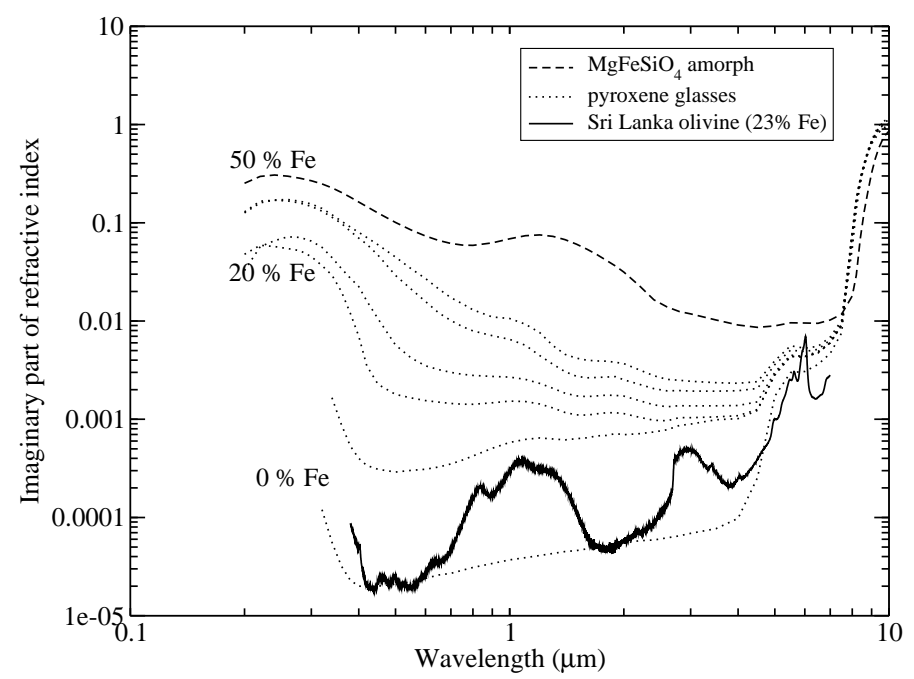

Figure 4: Vis/NIR absorption of Mg-Fe silicate glasses [14]] with various iron content compared to that of an iron-rich olivine crystal from Sri Lanka [67]. Absorption bands are due to crystal-field transitions (at 0.7$2 \mu \mathrm{m}$ ), water impurities (at $3 \mu \mathrm{m}$ ) and multi-phonon absorption (around $6 \mu \mathrm{m}$ ), whereas the background absorption throughout the vis/NIR is due to charge-transfer transitions involving ferric iron $\left(\mathrm{Fe}^{3+}\right)$.

composition containing iron see Jäger et al. [2]].

\section{2 vis/NIR absorption}

It is not obvious whether iron chemically bound in cosmic amorphous silicates would be in the ferrous oxidation state or not. Actually, the main effect of oxidized iron would concern the vis/NIR absorptivity of the silicate dust. Ferric iron causes a strong charge transfer transition absorption giving the glasses a brownish color, whereas ferrous iron only produces relatively weak crystal field bands in the near-infrared. Both absorption mechanisms are clearly seen in the optical constants of the Mg-Fe glasses (Fig. 团), while only the crystal field bands are present in the spectrum of an iron-rich olivine crystal [67]. Note that the absorption level in the visible differs by two orders of magnitude for a glass with $20 \%$ iron compared to the crystal with the same $\mathrm{Fe} / \mathrm{Mg}$ ratio. The total amount of iron in that glass is actually still much lower than in the olivine, because it is of pyroxene stoichiometry $((\mathrm{Mg}+\mathrm{Fe}) / \mathrm{Si}=1$ vs. $(\mathrm{Mg}+\mathrm{Fe}) / \mathrm{Si}=2$ for the crystal $)$.

Consequently, the absorptivity of silicate dust in the visible is a strong function not of the iron content in general but of the content of ferric iron. Metallic iron or iron sulfide present in inclusions would do a similarly good job [5]], but ferrous iron would not. Recent X-ray spectroscopic observations support the idea that most of the iron in interstellar space is in the metallic state, i.e. not oxidized at all ([[]], see also [प]). Ion irradiation could in fact be responsible for the separation of metallic iron phases from silicates (see also [[2]]) similarly as seen in GEMS [B]]. However, given the uncertainties both in the interpretation of infrared bands and in the fitting of the X-ray spectra, it is certainly still too early to conclude about the iron content of interstellar silicates.

\subsection{Sub-millimeter opacity}

At the far-infrared and sub-millimeter wavelengths, at which cold interstellar dust is thermally 


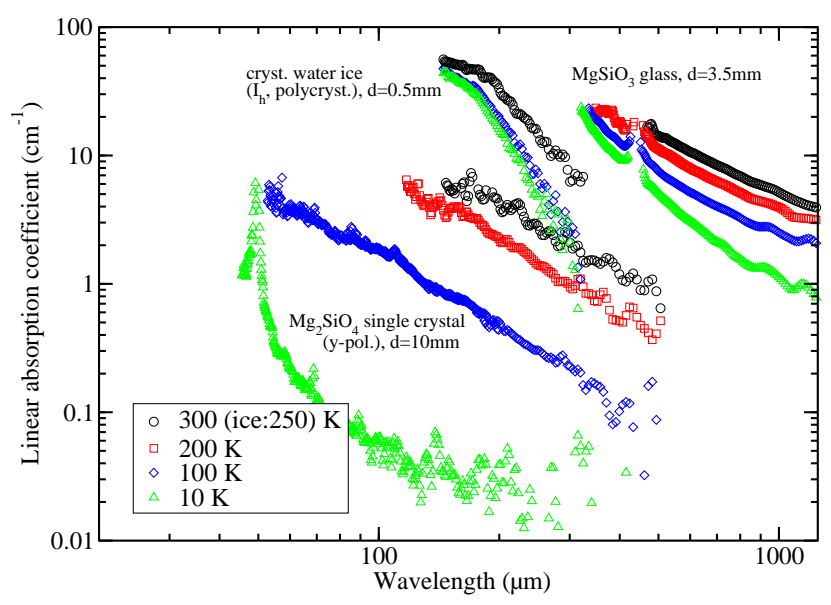

Figure 5: Far-infrared absorption coefficients measured on thick slabs of forsterite, crystalline water ice (pers. commun. C. Reinert), and $\mathrm{MgSiO}_{3}$ glass (pers. commun. P. Mohr) at temperatures between room temperature (highest values) and $10 \mathrm{~K}$ (lowest values). Note that reaching an optical depth of unity at 100 $\mu \mathrm{m}$ wavelength and $10 \mathrm{~K}$ requires a $20 \mathrm{~cm}$ long forsterite crystal, but only a few tens of microns thick glass.

emitting, amorphous silicates have an opacity which exceeds that of crystalline species considerably. This is illustrated in Fig. [1, where measured absorption coefficients of crystalline forsterite, crystalline water ice, and amorphous magnesium silicate are compared. All the absorption coefficients have been measured for compact slabs of material, i.e. the absolute values can be directly compared among each other but not to the absorption/emission cross section of particles without correction (see [49] for correction factors). All of the spectra show temperature dependencies and decrease towards longer wavelengths. The opacity of the amorphous silicate is the only one that could be measured at wavelengths exceeding $1 \mathrm{~mm}$ due to its large value.

One reason for the high opacity is the disorder-induced activation of phonons, another one is believed to be caused by low-energetic tunneling transitions of certain structural units in the amorphous network [4]]. Experimental opacity data for amorphous silicates are, however, still quite rare and poorly understood in the details of their dependence on composition and production method. Meanwhile, it is clear that they can be represented by power laws with single "spectral index" (i.e. negative exponent) $\beta$ only in limited spectral ranges. The urgent need for more systematic studies is illustrated by the mass absorption coefficient values compiled from various references in Table 1. At first glance the absolute $\kappa$ values appear neither correlated with the $\mathrm{Mg} / \mathrm{Si}$ ratio nor with the production method (sol-gel vs. melting). A possible influence of the iron content seems to be smaller than the spread of kappa values among the magnesium silicate data.

Strong efforts are currently underway by several groups to understand better the relation of the far-infrared spectra of amorphous silicates with their composition and structure (see [[13, [46]). Progress can be expected concerning the influence of iron and the extension of the data to larger wavelengths, which will be helpful to constrain the structure-dependent models as developed by Meny et al. [4] ] For this sake, it is inevitable to analyze the silicate structure by advanced analytical methods. Important issues are likely a characterization of the bonding state of the individual 
Table 1: Approximate mass absorption coefficients $\kappa$ (unit $\mathrm{cm}^{2} / \mathrm{g}$ ) and power law indices $\beta$ of amorphous silicates $(\mathrm{s} / \mathrm{g}=\mathrm{sol} / \mathrm{gel}$ products) from different references. Data are for isolated spherical particles in vacuum, i.e. have been corrected for the influences of agglomeration and embedding of the particles in the measurements. Data have been read from Mennella et al. [B8] , Boudet et al. [ $[\mathbf{D}]$, Coupeaud et al. [Q] , and Agladze et al. [四.

\begin{tabular}{|c|c|c|c|c|c|c|}
\hline & \multicolumn{3}{|c|}{ room temperature } & \multicolumn{3}{|c|}{$10-30 \mathrm{~K}$} \\
\hline & $\kappa(100 \mu \mathrm{m})$ & $\kappa(1 \mathrm{~mm})$ & $\beta(1 \mathrm{~mm})$ & $\kappa(100 \mu \mathrm{m})$ & $\kappa(1 \mathrm{~mm})$ & $\beta(1 \mathrm{~mm})$ \\
\hline am. Fe-Sil.[B]] & 112 & 5.0 & 1.35 & 94 & 0.86 & 2.04 \\
\hline $\mathrm{s} / \mathrm{g} \mathrm{MgSiO}_{3}[[]]$ & 50 & 0.98 & 1.44 & 40 & 0.12 & 2.7 \\
\hline glassy $\mathrm{MgSiO}_{3}[$ [] & 50 & 0.75 & 1.6 & 45 & 0.22 & 2.1 \\
\hline $\mathrm{s} / \mathrm{g} \mathrm{Mg}_{2} \mathrm{SiO}_{4}[\mathrm{~g}]$ & 250 & 6 & $1.6-2.2$ & 250 & 1.2 & $2.1-3.6$ \\
\hline s/g $\mathrm{MgSiO}_{3}[Q]$ & 200 & 10 & $1.7-0.9$ & 140 & 1.2 & $2.5-0.9$ \\
\hline $\mathrm{s} / \mathrm{g} \mathrm{MgSiO}_{3}$ [四] & & & & & 0.28 & 2.2 \\
\hline $\mathrm{s} / \mathrm{g} \mathrm{Mg}_{2} \mathrm{SiO}_{4}$ [四] & & & & & 0.20 & 2.2 \\
\hline
\end{tabular}

network-forming and -modifying atoms, but also of the medium-range order in the network (correlation of defects), as well as of the role of impurities (e.g., water, $\mathrm{OH}$ groups). Last but not least, the influence of the macroscopic structure should not be forgotten.

\section{Summary}

Optical measurements using laboratory analogs have provided useful data for modeling interstellar dust extinction and emission. However, in certain spectral ranges a full understanding of the optical properties of amorphous materials is not yet reached. These properties are related to the disordered structure of such materials on different length scales. For hydrogenated amorphous carbon, e.g., the medium-range structures and also the chemical bonding (by Raman and infrared spectroscopy) have been studied for a large diversity of analogs, and relations to spectral properties have been established. However, the particular structures that would produce certain observed features such as the UV extinction hump have not yet been resolved.

The disordered structure of amorphous silicates is similarly variable because different atoms can be accommodated that may be either network former or network modifier or both. The disorder on different length scales depends not only on the composition but also on the production process and will influence the optical properties in all spectral ranges. The visual absorption of amorphous silicates, e.g., depends primarily on the oxidation state (and presence) of iron, while the midinfrared bands depend on the bonding of all present atoms and the resulting polymerization state of the network. Least understood, so far, is the far-infrared and sub-millimeter opacity of amorphous silicates, but strong efforts are currently underway to investigate its dependence on short-range and medium-range structures in this important wavelength range. 
Progress in our understanding of interstellar dust will partially depend on the progress in understanding the structures of amorphous materials and their relation to the optical spectra in different wavelength ranges. Advanced analytical techniques to obtain structural information will play an important role in these efforts.

\section{Acknowledgement}

Laboratory astrophysics research at AIU Jena is supported by the German Research Foundation (DFG), particularly under the Priority Programmes SPP 1385 "The First 10 Mio. Years of the Solar System" and SPP 1573 "The Physics of the Interstellar Medium". We further acknowledge support for K.A.K. Gadallah by the Egyption Government.

\section{References}

[1] N. I. Agladze, A. J. Sievers, S. A. Jones, J. M. Burlitch, and S. V. W. Beckwith. Laboratory Results on Millimeter-Wave Absorption in Silicate Grain Materials at Cryogenic Temperatures. Astrophys. J., 462:1026, May 1996.

[2] N. Boudet, H. Mutschke, C. Nayral, C. Jäger, J.-P. Bernard, T. Henning, and C. Meny. Temperature Dependence of the Submillimeter Absorption Coefficient of Amorphous Silicate Grains. Astrophys. J., 633:272-281, November 2005.

[3] J. P. Bradley. Chemically Anomalous, Preaccretionally Irradiated Grains in Interplanetary Dust From Comets. Science, 265:925-929, August 1994.

[4] J. R. Brucato, G. Strazzulla, G. Baratta, and L. Colangeli. Forsterite amorphisation by ion irradiation: Monitoring by infrared spectroscopy. Astron. Astrophys., 413:395-401, January 2004.

[5] J. A. Cardelli, G. C. Clayton, and J. S. Mathis. The relationship between infrared, optical, and ultraviolet extinction. Astrophys. J., 345:245-256, October 1989.

[6] L. Colangeli, E. Bussoletti, V. Capozzi, and A. Minafra. Extinction spectra of amorphous carbon submicron grains in the UV-visible range. Astron. Astrophys., 168:349-353, November 1986.

[7] E. Costantini. The X-ray side of the absorption by interstellar dust in the Milky Way. In The Life Cycle of Dust in the Universe: Observations, Theory, and Laboratory Experiments, 2013)006, 2014.

[8] E. Costantini, C. Pinto, J. S. Kaastra, J. J. M. in't Zand, M. J. Freyberg, L. Kuiper, M. Méndez, C. P. de Vries, and L. B. F. M. Waters. XMM-Newton observation of 4U 1820-30. Broad band spectrum and the contribution of the cold interstellar medium. Astron. Astrophys., 539:A32, March 2012.

[9] A. Coupeaud, K. Demyk, C. Meny, C. Nayral, F. Delpech, H. Leroux, C. Depecker, G. Creff, J.-B. Brubach, and P. Roy. Low-temperature FIR and submillimetre mass absorption coefficient of interstellar silicate dust analogues. Astron. Astrophys., 535:A124, November 2011.

[10] K. L. Day. A possible identification of the 10-micron "silicate" feature. Astrophys. J. Lett., 192:L15-L17, August 1974.

[11] K. L. Day, T. R. Steyer, and D. R. Huffman. A Quantitative Study of Silicate Extinction. Astrophys. J., 191:415-418, July 1974. 
[12] K. Demyk, P. Carrez, H. Leroux, P. Cordier, A. P. Jones, J. Borg, E. Quirico, P. I. Raynal, and L. d'Hendecourt. Structural and chemical alteration of crystalline olivine under low energy $\mathrm{He}^{+}$ irradiation. Astron. Astrophys., 368:L38-L41, March 2001.

[13] K. Demyk and et al. FIR and Submm Optical Properties of Astrophysically Relevant Minerals. In The Life Cycle of Dust in the Universe: Observations, Theory, and Laboratory Experiments, POS (LCDU $2 0 1 3 \longdiv { 0 4 4 , 2 0 1 4 . }$

[14] J. Dorschner, B. Begemann, T. Henning, C. Jaeger, and H. Mutschke. Steps toward interstellar silicate mineralogy. II. Study of Mg-Fe-silicate glasses of variable composition. Astron. Astrophys., 300:503, August 1995.

[15] B. T. Draine. Interstellar Dust Models and Evolutionary Implications. In T. Henning, E. Grün, and J. Steinacker, editors, Cosmic Dust - Near and Far, volume 414 of Astronomical Society of the Pacific Conference Series, page 453, December 2009.

[16] E. L. Fitzpatrick. Correcting for the Effects of Interstellar Extinction. Publ. Astron. Soc. Pacific, 111:63-75, January 1999.

[17] E. L. Fitzpatrick and D. Massa. An Analysis of the Shapes of Interstellar Extinction Curves. V. The IR-through-UV Curve Morphology. Astrophys. J., 663:320-341, July 2007.

[18] K. A. K. Gadallah, H. Mutschke, and C. Jäger. UV irradiated hydrogenated amorphous carbon (HAC) materials as a carrier candidate of the interstellar UV bump at $217.5 \mathrm{~nm}$. Astron. Astrophys., 528:A56, April 2011.

[19] S. L. Hallenbeck, P. Daukantas, and J. A. Nuth. Mid-infrared spectral evolution of amorphous magnesium and iron silicate smokes due to vacuum annealing at $\mathrm{T}$ greater than or equal to $1000 \mathrm{~K}$. In Lunar and Planetary Institute Science Conference Abstracts, volume 28 of Lunar and Planetary Institute Science Conference Abstracts, page 497, March 1997.

[20] T. Henning and H. Mutschke. Low-temperature infrared properties of cosmic dust analogues. Astron. Astrophys., 327:743-754, November 1997.

[21] C. Jaeger, H. Mutschke, B. Begemann, J. Dorschner, and T. Henning. Steps toward interstellar silicate mineralogy. 1: Laboratory results of a silicate glass of mean cosmic composition. Astron. Astrophys., 292:641-655, December 1994.

[22] C. Jäger. Laboratory studies on dust condensation and processing in astrophysical environments. In The Life Cycle of Dust in the Universe: Observations, Theory, and Laboratory Experiments, POS (LCDU 2013) 045, 2014.

[23] C. Jäger, J. Dorschner, H. Mutschke, T. Posch, and T. Henning. Steps toward interstellar silicate mineralogy. VII. Spectral properties and crystallization behaviour of magnesium silicates produced by the sol-gel method. Astron. Astrophys., 408:193-204, September 2003.

[24] C. Jäger, D. Fabian, F. Schrempel, J. Dorschner, T. Henning, and W. Wesch. Structural processing of enstatite by ion bombardment. Astron. Astrophys., 401:57-65, April 2003.

[25] C. Jäger, F. Huisken, H. Mutschke, I. L. Jansa, and T. Henning. Formation of Polycyclic Aromatic Hydrocarbons and Carbonaceous Solids in Gas-Phase Condensation Experiments. Astrophys. J., 696:706-712, May 2009.

[26] C. Jäger, S. Krasnokutski, A. Staicu, F. Huisken, H. Mutschke, T. Henning, W. Poppitz, and I. Voicu. Identification and Spectral Properties of Polycyclic Aromatic Hydrocarbons in Carbonaceous Soot Produced by Laser Pyrolysis. Astrophys. J. Suppl. Ser., 166:557-566, October 2006. 
[27] C. Jager, H. Mutschke, and T. Henning. Optical properties of carbonaceous dust analogues. Astron. Astrophys., 332:291-299, April 1998.

[28] C. Jäger, H. Mutschke, T. Henning, and F. Huisken. Spectral Properties of Gas-phase Condensed Fullerene-like Carbon Nanoparticles from Far-ultraviolet to Infrared Wavelengths. Astrophys. J., 689:249-259, December 2008.

[29] C. Joblin, A. Leger, and P. Martin. Contribution of polycyclic aromatic hydrocarbon molecules to the interstellar extinction curve. Astrophys. J. Lett., 393:L79-L82, July 1992.

[30] A. P. Jones. Variations on a theme - the evolution of hydrocarbon solids. II. Optical property modelling - the optEC $(s)$ model. Astron. Astrophys., 540:A2, April 2012.

[31] C. Kaito, Y. Ojima, K. Kamitsuji, O. Kido, Y. Kimura, H. Suzuki, T. Sato, T. Nakada, Y. Saito, and C. Koike. Demonstration of crystalline forsterite grain formation due to coalescence growth of $\mathrm{Mg}$ and $\mathrm{SiO}$ smoke particles. Meteoritics and Planetary Science, 38:49-58, January 2003.

[32] C. Koike, H. Hasegawa, and A. Manabe. Extinction coefficients of amorphous carbon grains from 2100 A to 340 microns. Astrophys. Space Sci., 67:495-502, February 1980.

[33] C. Koike, C. Kaito, and H. Shibai. A Correlation Between the Microcrystallite Size and the Optical Properties of Amorphous Carbon Grains. Mon. Not. R. Astron. Soc., 268:321, May 1994.

[34] S. Kwok and Y. Zhang. Unidentified Infrared Emission Bands: PAHs or MAONs? Astrophys. J., 771:5, July 2013.

[35] I. Llamas-Jansa, C. Jäger, H. Mutschke, and Th. Henning. Far-ultraviolet to near-infrared optical properties of carbon nanoparticles produced by pulsed-laser pyrolysis of hydrocarbons and their relation with structural variations. CARBON, 45(7):1542-1557, JUN 2007.

[36] V. Mennella. Activation Energy of $\mathrm{CH}$ Bond Formation in Carbon Grains Irradiated with Hydrogen Atoms. Astrophys. J. Lett., 647:L49-L52, August 2006.

[37] V. Mennella, G. A. Baratta, A. Esposito, G. Ferini, and Y. J. Pendleton. The Effects of Ion Irradiation on the Evolution of the Carrier of the 3.4 Micron Interstellar Absorption Band. Astrophys. J., 587:727-738, April 2003.

[38] V. Mennella, J. R. Brucato, L. Colangeli, P. Palumbo, A. Rotundi, and E. Bussoletti. Temperature Dependence of the Absorption Coefficient of Cosmic Analog Grains in the Wavelength Range 20 Microns to 2 Millimeters. Astrophys. J., 496:1058, March 1998.

[39] V. Mennella, L. Colangeli, P. Palumbo, A. Rotundi, W. Schutte, and E. Bussoletti. Activation of an Ultraviolet Resonance in Hydrogenated Amorphous Carbon Grains by Exposure to Ultraviolet Radiation. Astrophys. J. Lett., 464:L191, June 1996.

[40] V. Mennella, G. M. Muñoz Caro, R. Ruiterkamp, W. A. Schutte, J. M. Greenberg, J. R. Brucato, and L. Colangeli. UV photodestruction of $\mathrm{CH}$ bonds and the evolution of the $3.4 \mathrm{mu}$ feature carrier. II. The case of hydrogenated carbon grains. Astron. Astrophys., 367:355-361, February 2001.

[41] C. Meny, V. Gromov, N. Boudet, J.-P. Bernard, D. Paradis, and C. Nayral. Far-infrared to millimeter astrophysical dust emission. I. A model based on physical properties of amorphous solids. Astron. Astrophys., 468:171-188, June 2007.

[42] E. R. Micelotta, A. P. Jones, J. Cami, E. Peeters, J. Bernard-Salas, and G. Fanchini. The Formation of Cosmic Fullerenes from Arophatic Clusters. Astrophys. J., 761:35, December 2012.

[43] P. S. Miedema and F. M. F. de Groot. The iron L edges: Fe 2p X-ray absorption and electron energy loss spectroscopy. J. Electron Spectrosc. Rel. Phenom., 187:32-48, March 2013. 
[44] M. Min. The appearance of large aggregate particles in cosmic environments. In The Life Cycle of Dust in the Universe: Observations, Theory, and Laboratory Experiments, POS (LCDU 2013) 038 , 2014.

[45] M. Min, L. B. F. M. Waters, A. de Koter, J. W. Hovenier, L. P. Keller, and F. Markwick-Kemper. The shape and composition of interstellar silicate grains. Astron. Astrophys., 462:667-676, February 2007.

[46] P. Mohr, H. Mutschke, and F. Lewen. Laboratory measurements of the far-infrared to millimeter dust opacity of amorphous $\mathrm{Mg} / \mathrm{Fe}$ silicates. In The Life Cycle of Dust in the Universe: Observations, Theory, and Laboratory Experiments, POS (LCDU 2013) 140, 2014.

[47] H. Mutschke, B. Begemann, J. Dorschner, J. Guertler, B. Gustafson, T. Henning, and R. Stognienko. Steps toward interstellar silicate mineralogy. III. The role of aluminium in circumstellar amorphous silicates. Astron. Astrophys., 333:188-198, May 1998.

[48] H. Mutschke, M. Min, and A. Tamanai. Laboratory-based grain-shape models for simulating dust infrared spectra. Astron. Astrophys., 504:875-882, September 2009.

[49] H. Mutschke, S. Zeidler, and H. Chihara. Far-infrared continuum absorption of olivine at low temperatures. Earth, Planets, and Space, 65:1139-1143, October 2013.

[50] V. Ossenkopf, T. Henning, and J. S. Mathis. Constraints on cosmic silicates. Astron. Astrophys., 261:567-578, August 1992.

[51] R. Papoular, J. Conard, O. Guillois, I. Nenner, C. Reynaud, and J.-N. Rouzaud. A comparison of solid-state carbonaceous models of cosmic dust. Astron. Astrophys., 315:222-236, November 1996.

[52] Y. J. Pendleton and L. J. Allamandola. The Organic Refractory Material in the Diffuse Interstellar Medium: Mid-Infrared Spectroscopic Constraints. Astrophys. J. Suppl. Ser., 138:75-98, January 2002.

[53] T. Sabri, L. Gavilan, C. Jäger, J. L. Lemaire, G. Vidali, H. Mutschke, and T. Henning. Interstellar Silicate Analogs for Grain-surface Reaction Experiments: Gas-phase Condensation and Characterization of the Silicate Dust Grains. Astrophys. J., 780:180, January 2014.

[54] A. Sakata, S. Wada, Y. Okutsu, H. Shintani, and Y. Nakada. Does a 2,200 A hump observed in an artificial carbonaceous composite account for UV interstellar extinction. Nature, 301:493, February 1983.

[55] M. Schnaiter, T. Henning, H. Mutschke, B. Kohn, M. Ehbrecht, and F. Huisken. Infrared Spectroscopy of Nano-sized Carbon Grains Produced by Laser Pyrolysis of Acetylene: Analog Materials for Interstellar Grains. Astrophys. J., 519:687-696, July 1999.

[56] M. Schnaiter, H. Mutschke, J. Dorschner, T. Henning, and F. Salama. Matrix-isolated Nano-sized Carbon Grains as an Analog for the 217.5 Nanometer Feature Carrier. Astrophys. J., 498:486, May 1998.

[57] A. Scott and W. W. Duley. Ultraviolet and Infrared Refractive Indices of Amorphous Silicates. Astrophys. J. Suppl. Ser., 105:401, August 1996.

[58] A. K. Speck, A. G. Whittington, and A. M. Hofmeister. Disordered Silicates in Space: A Study of Laboratory Spectra of "Amorphous" Silicates. Astroph. J., 740:93, October 2011.

[59] M. Steglich, Y. Carpentier, C. Jäger, F. Huisken, H.-J. Räder, and T. Henning. The smoothness of the interstellar extinction curve in the UV. Comparison with recent laboratory measurements of PAH mixtures. Astron. Astrophys., 540:A110, April 2012. 
[60] M. Steglich, F. Huisken, J. E. Dahl, R. M. K. Carlson, and T. Henning. Electronic Spectroscopy of FUV-irradiated Diamondoids: A Combined Experimental and Theoretical Study. Astrophys. J., 729:91, March 2011.

[61] M. Steglich, C. Jäger, G. Rouillé, F. Huisken, H. Mutschke, and T. Henning. Electronic Spectroscopy of Medium-sized Polycyclic Aromatic Hydrocarbons: Implications for the Carriers of the $2175 \AA$ UV Bump. Astrophys. J. Lett., 712:L16-L20, March 2010.

[62] R. Stognienko, T. Henning, and V. Ossenkopf. Optical properties of coagulated particles. Astron. Astrophys., 296:797, April 1995.

[63] S. P. Thompson. Structural signatures of medium-range order in annealed laboratory silicates. Astron. Astrophys., 484:251-265, June 2008.

[64] S. P. Thompson, S. Fonti, C. Verrienti, A. Blanco, V. Orofino, and C. C. Tang. Laboratory study of annealed amorphous $\mathrm{MgSiO}_{3}$ silicate using IR spectroscopy and synchrotron X-ray diffraction. Astron. Astrophys., 395:705-717, November 2002.

[65] S. P. Thompson, J. E. Parker, and C. C. Tang. The $10 \mu \mathrm{m}$ band in amorphous $\mathrm{MgSiO}_{3}$ : the influence of medium-range structure, defects and thermal processing. Astron. Astrophys., 545:A60, September 2012.

[66] D. Virgo and B. O. Mysen. The structural state of iron in oxidized vs. reduced glasses at 1 atm: $\mathrm{A}^{57} \mathrm{Fe}$ Mössbauer study. Phys. Chem. Minerals, 12:65-76, March 1985.

[67] S. Zeidler, T. Posch, H. Mutschke, H. Richter, and O. Wehrhan. Near-infrared absorption properties of oxygen-rich stardust analogs. The influence of coloring metal ions. Astron. Astrophys., 526:A68, February 2011.

[68] V. G. Zubko, V. Mennella, L. Colangeli, and E. Bussoletti. Optical constants of cosmic carbon analogue grains - I. Simulation of clustering by a modified continuous distribution of ellipsoids. Mon. Not. R. Astron. Soc., 282:1321-1329, October 1996. 